\title{
Consensus Conditions for High-Order Multiagent Systems with Nonuniform Delays
}

\author{
Mengji Shi, Kaiyu Qin, Ping Li, and Jun Liu \\ School of Aeronautics and Astronautics, University of Electronic Science and Technology of China, Chengdu 611731, China \\ Correspondence should be addressed to Kaiyu Qin; kyqin@uestc.edu.cn
}

Received 20 May 2016; Revised 8 October 2016; Accepted 16 October 2016; Published 9 April 2017

Academic Editor: Guido Ala

Copyright (C) 2017 Mengji Shi et al. This is an open access article distributed under the Creative Commons Attribution License, which permits unrestricted use, distribution, and reproduction in any medium, provided the original work is properly cited.

\begin{abstract}
Consensus of first-order and second-order multiagent systems has been wildly studied. However, the convergence of high-order (especially the third-order to the sixth-order) state variables is also ubiquitous in various fields. The paper handles consensus problems of high-order multiagent systems in the presence of multiple time delays. Obtained by a novel frequency domain approach which properly resolves the challenges associated with nonuniform time delays, the consensus conditions for the first-order and second-order systems are proven to be nonconservative, and those for the third-order to the sixth-order systems are provided in the form of simple inequalities. The method revealed in this article is applicable to arbitrary-order systems, and the results are less conservative than those based on Lyapunov approaches, because it roots in sufficient and necessary criteria of stabilities. Simulations are carried out to validate the theoretical results.
\end{abstract}

\section{Introduction}

Consensus problems of multiagent systems have found many applications in the fields that hold great promise, including (but not limited to) biosciences, robotics, and computer sciences. Consensus is the agreement regarding a certain quality of interest on specific states of all the agents, which is widely demanded in the engineering applications. The research on consensus problems has lasted for decades. Various techniques are developed to solve consensus problems of numerous multiagent systems [1-23].

This paper addresses the consensus control problems of high-order multiagents systems with nonuniform time delays. One motivation for studying high-order systems is to achieve accurate control of complex motion: for example, when performing consensus motion that requires abrupt change of heading, a team of vehicles should maintain consistency of acceleration (as well as position and velocity) among them by controlling the third-order state (acceleration), while lower-order (first-order and second-order) consensus protocols are usually designed for more regular motion (e.g., rectilinear [16] and rotational [23] motion). Besides highorder dynamics [3-9], nonlinearities [1, 2, 24], time delays $[8-21,24,25]$ and fuzziness $[26,27]$ also bring complexities to the control systems, which often lead to difficulties in stability analysis.

A novel frequency-domain-based method is developed to challenge the system complexities and derive the consensus conditions. Comparing to the universal stability analysis tool Lyapunov approaches, frequency domain methods are more possibly conducing to less conservative results as it roots in sufficient and necessary stability criteria. On the other hand, Lyapunov approaches applied in many literatures yield consensus conditions in the form of Linear Matrix Inequalities (LMIs) [7-9], while with frequency domain methods authors of $[12-16,22]$ as well as this note obtain consensus conditions in the form of inequalities which are more perspicuous and simple to calculate. However, frequency domain methods are limited to linear and timeinvariant systems, and consequently most of the aforementioned articles [1-11, 24, 25] especially those coping with nonlinear and high-order systems have adopted Lyapunov 
approaches instead. Moreover, both high-order dynamics and nonuniform time delays give rise to dramatic increment of the systems' dimensionality, which makes it a knotty problem. In existing literatures, methods based on the properties of nonnegative matrices [17, 18] and Nyquist stability theories [19-21] are introduced as alternative stability analysis tools.

The main idea of the proposed approach is to transform the high-order systems' dynamics into high-degree polynomials with respect to hypothetically existing imaginary eigenvalues of the systems. By studying the monotonicity of the polynomials and their derivatives, consensus conditions can be figured out in the form of inequalities. The present work first brings out sufficient consensus conditions for the first-order to sixth-order nonuniformly delayed systems which are most likely to apply to practical engineering applications [28]: if all the delays are bounded by a given value and all the parameters agree to corresponding inequalities, the systems can achieve consensus and in addition, for the sake of nonconservativeness, the paper provides stronger conditions for the first-order and second-order systems by thorough derivation: the states converge when all the delays are bounded by a given value but diverge when all the delays exceed that value.

Literature [29] has proposed a high-order nonlinear consensus tracking algorithm with unmeasurable system states which applies to wide-range multiagent systems and proven the achievement of consensus by constructing Lyapunov functions, while time delays are not considered. In [9], Zhang et al. have solved average consensus problem of high-order multiagent system with time-varying delays and provided stability conditions in the form of LMIs via a LyapunovKrasovskii approach. The authors of [19] have derived necessary and sufficient consensus conditions for large-scale high-order linear multiagent systems with heterogeneous communication delays by using the generalized Nyquist criterion; however, the derived consensus conditions are setvalued graphical conditions, and inequality conditions are only derived for the first-order system. The previous work in [30] has studied consensus motion of delayed second-order multiagent systems by a nonconservative frequency-domainbased method, while this article will present stationary consensus conditions for more complex high-order multiagent systems.

The remainder of this note is organized as follows: Section 2 states the consensus problem with the help of graph theory; Section 3 presents the main results by demonstrating the stability analysis; Section 4 depicts the selected simulation experiments; Section 5 draws conclusions with future research directions.

\section{Problem Statement}

This section starts with some definitions and results in graph theory.

Consider an $n$-agent system. The communication network topology among them is represented by an undirected graph $\mathscr{G}(\mathscr{V}, \mathscr{E}, \mathscr{A})$, which consists of a set of nodes $\mathscr{V}=\left\{s_{i}\right\}$, $i \in \mathscr{I}=\{1,2, \ldots, n\}$, a set of edges $\mathscr{E} \subseteq \mathscr{V} \times \mathscr{V}$, and a weighted adjacency matrix $\mathscr{A}=\left[a_{i j}\right]$, where $a_{i i}=0$ and $a_{i j}=a_{j i} \geq 0$ (for $\mathscr{G}$ is undirected). $a_{i j}>0$ if and only if there exists an edge $e_{i j} \in \mathscr{E}$ between the $i$ th and $j$ th nodes, which implies that they can get information from each other. The set of neighbors of node $s_{i}$ is denoted by $N_{i} \triangleq\left\{s_{j} \in \mathscr{V}: e_{j i} \in \mathscr{E}\right\}$. The Laplacian corresponding to the graph $\mathscr{G}$ is defined as $L_{\sigma} \triangleq\left[\ell_{i j}\right]$, where $\ell_{i i}=\sum_{j=1}^{n} a_{i j}$ and $\ell_{i j}=-a_{i j}, i \neq j$. A path is a sequence of indexed edges $e_{k_{1} k_{2}}, e_{k_{2} k_{3}}, \ldots$, where $e_{k_{i} k_{i+1}} \in \mathscr{E}$. If there is a path between every pair of nodes in graph $\mathscr{G}$, the graph is said to be connected. The following lemma is given by [31].

Lemma 1. If the undirected graph $\mathscr{G}$ is connected, then its Laplacian $L_{\sigma}$ has one singleton zero eigenvalue (with eigenvector 1), and the rest $n-1$ eigenvalues of $L_{\sigma}$ are all positive.

Consider an $l$ th-order multiagent system consisting of $n$ agents. The dynamics of the $i$ th agent $(i \in \mathscr{I})$ is

$$
\begin{gathered}
\dot{\psi}_{i}^{(0)}(t)=\psi_{i}^{(1)}(t), \\
\vdots \\
\dot{\psi}_{i}^{(l-2)}(t)=\psi_{i}^{(l-1)}(t), \\
\dot{\psi}_{i}^{(l-1)}(t)=u_{i}(t),
\end{gathered}
$$

where $\psi_{i}^{(k)} \in \mathbb{R}$ is the $k$ th state variable of the $i$ th agent, $k=$ $0,1, \ldots, l-1$, and $u_{i}(t) \in \mathbb{R}$ is the control input. Let $\psi_{i} \triangleq$ $\left[\psi_{i}^{(0)}, \psi_{i}^{(1)}, \ldots, \psi_{i}^{(l-1)}\right]^{T}$ be the state vector of the $i$ th agent; we assume that the initial conditions are $\psi_{i}^{(0)}(s)=\psi_{i}^{(0)}(0)$ and $\psi_{i}^{(k)}(s)=\psi_{i}^{(k)}(0)=0, k=1,2, \ldots, l-1$, for $s \in(-\infty, 0]$. The control input $u_{i}(t)$ is said to solve the consensus problem asymptotically, if and only if $\lim _{t \rightarrow+\infty}\left[\psi_{i}(t)-\psi_{j}(t)\right]=0$ for all $i, j \in \mathscr{I}$.

In [8], a discrete-time control input was introduced as

$$
\begin{aligned}
u_{i}(k)= & -\sum_{j=1}^{l-1} p_{j} \psi_{i}^{(j)}(k) \\
& -\sum_{s_{j} \in N_{i}(k)} a_{i j}(k)\left[\psi_{i}^{(0)}(k)-\psi_{j}^{(0)}\left(k-\tau_{i j}\right)\right] .
\end{aligned}
$$

In this paper, we introduce an continuous-time consensus algorithm for system (1) with multiple time delays, and the input delays are supposed to occur. The protocol is

$$
\begin{aligned}
u_{i}(t)= & -\sum_{j=1}^{l-1} p_{j} \psi_{i}^{(j)}(t) \\
& -\sum_{s_{j} \in N_{i}} a_{i j}\left[\psi_{i}^{(0)}\left(t-\tau_{i j}\right)-\psi_{j}^{(0)}\left(t-\tau_{i j}\right)\right],
\end{aligned}
$$

for any $i \in \mathscr{I}$, where $p_{j}>0$ for $j=1,2, \ldots, l-1 ; a_{i j}>0$ denotes the edge weight, and $\tau_{i j}=\tau_{j i}$ is the time delay for the $i$ th agent to get the state information of the $j$ th agent. We assume that the system has $M$ different time delays, denoted by $\tau_{m} \in\left(\tau_{i j}, i, j \in \mathscr{I}(m=1,2, \ldots, M)\right.$. 
Let $\psi(t) \triangleq\left[\psi_{1}(t), \psi_{2}(t), \ldots, \psi_{n}(t)\right]$, and

$$
\begin{aligned}
A \triangleq\left[\begin{array}{ccccc}
0 & 1 & 0 & 0 & 0 \\
0 & 0 & 1 & 0 & 0 \\
\vdots & \ddots & \ddots & \ddots & 0 \\
0 & \vdots & 0 & 0 & 1 \\
0 & -p_{1} & \ldots & -p_{l-2} & -p_{l-1}
\end{array}\right] \in \mathbb{R}^{l \times l}, \\
B \triangleq\left[\begin{array}{cccc}
0 & 0 & \ldots & 0 \\
\vdots & \vdots & \vdots & \vdots \\
0 & 0 & \ldots & 0 \\
1 & 0 & \ldots & 0
\end{array}\right] \in \mathbb{R}^{l \times l} .
\end{aligned}
$$

Under the control input given by (3), the network dynamics of the multiagent system becomes

$$
\dot{\psi}(t)=\left(I_{n} \otimes A\right) \psi(t)-\sum_{m=1}^{M}\left(L_{\sigma m} \otimes B\right) \psi\left(t-\tau_{m}\right)
$$

with the initial condition $\psi(s)=\psi(0), s \in(-\infty, 0]$, where $L_{\sigma m}$ denotes the Laplacian of a subgraph associated with the delay $\tau_{m}$. Clearly, $L_{\sigma}=\sum_{m=1}^{M} L_{\sigma m}$. If all the time delays are equal to zero, system (5) could be rewritten as

$$
\dot{\psi}(t)=\left(I_{n} \otimes A-L_{\sigma} \otimes B\right) \psi(t) .
$$

This paper assumes that the graph $\mathscr{G}$ is always connected and undirected.

\section{Main Results}

The following lemma presents a sufficient condition for the stability of high-degree polynomials given by [32], which is helpful in the present work.

Lemma 2. Consider a polynomial $f(x)=a_{0}+a_{1} x+\cdots+$ $a_{n} x^{n}$, where $a_{k}>0, k=0,1, \ldots, n, n \geq 3$, with coefficients of determination defined as

$$
\mu_{i} \triangleq \frac{a_{i-1} a_{i+2}}{a_{i} a_{i+1}},
$$

if all the coefficients of determination satisfy that $\mu_{i}<\beta / 2 \approx$ 0.4655 , where $i=1,2, \ldots, n-2, n \geq 3$, and $\beta$ is the only real root of equation

$$
\frac{\beta^{3}}{4}+\beta^{2}+\beta-2=0
$$

then all the roots of $f(x)=0$ have negative real parts.

Let

$$
\Phi \triangleq I_{n} \otimes A-L_{\sigma} \otimes B
$$

and suppose that the eigenvalues of $L_{\sigma}$ are $0=\lambda_{1}<\lambda_{2} \leq$ $\lambda_{3} \leq \cdots \leq \lambda_{n}$ according to Lemma 1 .
Assumption 3. Assume for $l \geq 3$ that all $p_{j}>0(j=$ $1,2, \ldots, l-1)$ satisfy the following:

$$
\begin{gathered}
p_{l-1} p_{l-2}>3 p_{l} p_{l-3} \\
p_{l-2} p_{l-3}>3 p_{l-1} p_{l-4} \\
\vdots \\
p_{3} p_{2}>3 p_{4} p_{1} \\
p_{2} p_{1}>2.15 p_{3} \lambda_{n},
\end{gathered}
$$

where $p_{l}=1$ and $\lambda_{n}$ is the largest eigenvalue of $L_{\sigma}$.

Under Assumption 3, the following lemma can be proven.

Lemma 4. Matrix $\Phi$ has a singleton zero eigenvalue and all other eigenvalues have negative real parts if Assumption 3 is satisfied.

Proof. According to Lemma 1, there exists an orthogonal matrix $W$, such that

$$
W^{T} L_{\sigma} W=\operatorname{diag}\left\{0, \lambda_{2}, \ldots, \lambda_{n}\right\},
$$

and then it follows that

$$
\begin{aligned}
(W & \left.\otimes I_{l}\right)^{T} \Phi\left(W \otimes I_{l}\right) \\
& =\operatorname{diag}\left\{A, A-\lambda_{2} B, \ldots, A-\lambda_{n} B\right\} .
\end{aligned}
$$

Through simple calculations, the eigenpolynomial of $(A-$ $\left.\lambda_{j} B\right)$ could be obtained; then we get

$$
\operatorname{det}\left(s I_{2}-A+\lambda_{j} B\right)=s^{l}+\sum_{i=1}^{l-1} p_{i} s^{i}+\lambda_{j}=0 .
$$

To simplify the following statements, let $p_{l}=1$, and (13) could be written as

$$
\sum_{i=1}^{l} p_{i} s^{i}+\lambda_{j}=0
$$

For the first-order system, since $l=1$, it is evident that $-\lambda_{j}$ are the eigenvalues of the system; thus the lemma is proven. For the second-order system, (14) becomes $s^{2}+p_{1} s+\lambda_{j}=0$ and its roots are

$$
\frac{-p_{1} \pm \sqrt{p_{1}^{2}-4 \lambda_{j}}}{2} .
$$

If $p_{1}^{2}<4 \lambda_{j}$, the real part of (15) is $-p_{1} / 2<0$, and when $p_{1}^{2} \geq$ $4 \lambda_{j},(15)$ apparently is a pair of nonpositive real numbers, and the bigger one equals zero if and only if $\lambda_{j}=0$; then the lemma is proven.

When $l \geq 3$, note that $p_{i}>0$ for $i=1,2, \ldots, l$, and $\lambda_{n}$ is the largest eigenvalue of $L_{\sigma}$. It is apparent that $3>2.15>$ $2.1482 \approx 1 / 0.4655$; according to Lemma $2,(10)$ is a group of more conservative condition, which ensures that all the roots of (14) have negative real parts except that there exists one singleton zero root for $\lambda_{j}=0$. Thus, matrix $\Phi$ has a singleton zero eigenvalue and all its other $n * l-1$ eigenvalues have negative real parts. 
Remark 5. Lemma 4 has shown that each of the nondelayed multiagent systems given by (6) has all its eigenvalues on the open LHP except one equals zero. That implies that system (6) is stable, and all the states of each agent will reach a common value. The existence of the only zero root indicates that only the first-order state variable of each agent reaches a value that is decided by the initial state and all the other high-order state variables return to zero at last; that is, if all $\tau_{m}=0$, multiagent system (5) will reach consensus.

By analyzing the effect of nonuniform time delays on the stability of the systems, we will give a proof to the ensuing theorem.

Theorem 6. Consider lth-order $(l=1,2, \ldots, 6)$ system given by (5) that satisfies Assumption 3, and the following inequalities (16), (17), and (18) are satisfied for $l=4,5,6$, respectively:

$$
\begin{aligned}
& p_{2}>\frac{\lambda_{n}}{\sqrt{p_{3} p_{1}}}+2 \frac{p_{1}}{p_{3}} . \\
& p_{1}>\frac{1}{4}\left(\frac{p_{2}}{p_{4}}\right)^{2} \\
& p_{1}>\max \left\{\frac{p_{5}}{4}\left(\frac{p_{2}}{p_{4}}\right)^{2}, \lambda_{n} \sqrt{\frac{3}{p_{4}}}\right\} .
\end{aligned}
$$

Define functions $\theta_{l}(\omega)$ and $T_{l}(\omega)(l=1,2, \ldots, 6)$ as follows:

$$
\begin{aligned}
\theta_{l}(\omega) & \triangleq \arg \left[F_{l}(\omega)\right]=\arg \left[-\sum_{i=1}^{l}(-j \omega)^{i} p_{i}\right] \\
& \in(0,2 \pi], \\
T_{l}(\omega) & \triangleq \frac{1}{\omega} \theta_{l}(\omega),
\end{aligned}
$$

where $F_{l}(\omega)$ is defined in (27). If all $\tau_{m}$ satisfy $\tau_{m}<\bar{\tau}$ for lthorder multiagent system, where

$$
\begin{aligned}
& \bar{\tau} \\
& = \begin{cases}T_{l}\left(\lambda_{n}\right) & l=1 \\
T_{l}\left(\sqrt{\lambda_{n} / p_{2}}\right) & l=2,3 \\
T_{l}\left(\sqrt{2 \lambda_{n} / p_{2}}\right) & l=4 \\
\min \left\{T_{l}\left(\sqrt{2 \lambda_{n} / p_{2}}\right), T_{l}\left(\sqrt{p_{l-2} /(l-3)}\right)\right\} & l=5,6,\end{cases}
\end{aligned}
$$

then control input (3) can solve the consensus problem of system (5).

Proof. Consider the network of high-order multiagents with nonuniform time delays. Let $\Psi(s)=G_{\tau}^{-1} \Psi(0)$, where $\Psi(s)$ is the Laplace transform of $\psi(t)$, and

$$
G_{\tau}(s)=s I_{n \times l}-\left(I_{n} \otimes A\right)+\sum_{m=1}^{M}\left(L_{\sigma m} \otimes B\right) e^{-\tau_{m} s} .
$$

According to the foregoing discussions, to study the stability of the delayed system, we only need to investigate the values of $\tau_{m}$ that guarantee the existence of nonzero roots of $G_{\tau}(s)$ on imaginary axis, which represents the crossing of the characteristic roots from the stable region to the unstable one. The roots of characteristic polynomials such as $G_{\tau}(s)$ are hereinafter referred to as "the eigenvalues of the system."

Suppose $s=j \omega \neq 0$ is an imaginary root of $G_{\tau}(s)$, and $u=u_{1} \otimes[1,0, \ldots, 0]^{T}+u_{2} \otimes[0,1, \ldots, 0]^{T}+\cdots+u_{l} \otimes[0,0, \ldots, 1]^{T}$ is a corresponding eigenvector, where $\|u\|=1, u_{i} \in \mathbb{C}^{n}, i=$ $1,2, \ldots, n$. Then we have

$$
\left[j \omega I_{n \times l}-\left(I_{n} \otimes A\right)+\sum_{m=1}^{M}\left(L_{\sigma m} \otimes B\right) e^{-j \omega \tau_{m}}\right] u=0 .
$$

Note that all the complex roots of each $G_{\tau}(s)$ appeared in conjugated pairs; we only need to study the situation that $\omega_{q}>0$. Since all of the first $l-1$ elements of the vector obtained by calculating the left part of (23) are equal to zero, we get

$$
j \omega u_{i}=u_{i+1}
$$

for all $i=1,2, \ldots, l-1$. Multiplied by $u^{*}$ (the conjugate transpose of $u$ ) on the left side of the left part of (23), and with (24) substituted, we obtain

$$
\sum_{m=1}^{M} \alpha_{m} e^{-j \omega \tau_{m}}+\sum_{i=1}^{l}(j \omega)^{i} p_{i}=0,
$$

where $p_{l}=1$, and

$$
\alpha_{m}=\frac{u^{*}\left(L_{\sigma m} \otimes I_{l}\right) u}{u^{*} u} .
$$

Rewrite (25) as

$$
\sum_{m=1}^{M} \alpha_{m} e^{j \omega \tau_{m}}=-\sum_{i=1}^{l}(-j \omega)^{i} p_{i} \triangleq F_{l}(\omega) .
$$

Take modulus of both sides of (27); then, we have

$$
\begin{aligned}
M_{l}(\omega) & \triangleq\left|F_{l}(\omega)\right|=\left|\sum_{m=1}^{M} \alpha_{m} e^{j \omega \tau_{m}}\right| \leq\left|\sum_{m=1}^{M} \alpha_{m}\right| \\
& =\frac{u^{*}\left(L_{\sigma} \otimes I_{l}\right) u}{u^{*} u} \leq \lambda_{n} .
\end{aligned}
$$

Another necessary condition of (27) is

$$
\arg \left(\sum_{m=1}^{M} \alpha_{m} e^{j \omega \tau_{m}}\right)=\arg \left[F_{l}(\omega)\right]=\theta_{l}(\omega) .
$$

As $\omega \tau_{m}>0, \theta_{l}(\omega)$ should be discussed in the positive interval $(0,2 \pi]$. From $(29)$, it is obvious that

$$
\theta_{l}(\omega)=\arg \left(\sum_{m=1}^{M} \alpha_{m} e^{j \omega \tau_{m}}\right) \leq \max \left\{\omega \tau_{m}\right\} .
$$

Define

$$
R_{l}(\omega) \triangleq \frac{\operatorname{Im}\left[F_{l}(\omega)\right]}{\operatorname{Re}\left[F_{l}(\omega)\right]}=\tan \left[\theta_{l}(\omega)\right]
$$


where $\operatorname{Im}(c)$ and $\operatorname{Re}(c)$ denote the imaginary part and real part of the complex number $c$, respectively. $R_{l}(\omega)$ is an access to $\theta_{l}(\omega)$.

Consider the first-order system; then, we have $F_{1}(\omega)=$ $0+j \omega$ and $R_{1}(\omega)=\omega / 0$. It is apparent that $\theta_{1}(\omega)=\pi / 2$, and $M_{1}(\omega)=\omega$; according to (28), we should only consider $\omega \leq \lambda_{n}$; if we set all $\tau_{m}<\bar{\tau}=T_{1}\left(\lambda_{n}\right)=\pi /\left(2 \lambda_{n}\right)$, then

$$
\omega \tau_{m}<\lambda_{n} \bar{\tau}=\pi / 2=\theta_{1}(\omega)
$$

$$
\begin{aligned}
& R_{2}^{\prime}=\frac{-p_{1} p_{2}}{\left(p_{2} \omega\right)^{2}}<0, \\
& R_{3}^{\prime}=\frac{-p_{2} p_{3} \omega^{2}-p_{1} p_{2}}{\left(p_{2} \omega\right)^{2}}<0, \\
& R_{4}^{\prime}=\frac{-p_{3} p_{4} \omega^{4}-\left(p_{2} p_{3}-3 p_{1} p_{4}\right) \omega^{2}-p_{1} p_{2}}{\left(p_{2} \omega-p_{4} \omega^{3}\right)^{2}}<0, \\
& R_{5}^{\prime}=\frac{-p_{4} p_{5} \omega^{6}-\left(p_{3} p_{4}-3 p_{2} p_{5}\right) \omega^{4}-\left(p_{2} p_{3}-3 p_{1} p_{4}\right) \omega^{2}-p_{1} p_{2}}{\left(p_{2} \omega-p_{4} \omega^{3}\right)^{2}}<0, \\
& R_{6}^{\prime}=\frac{-p_{5} p_{6} \omega^{8}-\left(p_{4} p_{5}-3 p_{3} p_{6}\right) \omega^{6}-\left(p_{3} p_{4}-3 p_{2} p_{5}+5 p_{1} p_{6}\right) \omega^{4}-\left(p_{2} p_{3}-3 p_{1} p_{4}\right) \omega^{2}-p_{1} p_{2}}{\left(p_{2} \omega-p_{4} \omega^{3}\right)^{2}}<0 .
\end{aligned}
$$

which contradicts (30). Then when all $\tau_{m}<\bar{\tau}$, the first-order system is impossible to have an imaginary eigenvalue which presents the first contact of the eigenvalues from the stable region to the unstable one. Hence the system is still stable then, and it can reach consensus, and the theorem is proven for $l=1$.

Unlike $\theta_{1}(\omega), \theta_{l}(\omega)(l \geq 2)$ are not a fixed value. But we have found that the derivatives of $R_{l}(\omega)(2 \leq l \leq 6)$ listed below are negative values when Assumption 3 is applied:
$R_{l}^{\prime}<0$ means that $R_{l}=\tan \left[\theta_{l}(\omega)\right]$ are monotonically decreasing with the growth of $\omega$. Then it can be deduced that the arguments $\theta_{l}(\omega)$ also decrease monotonically and continuously because the values of $F_{l}(\omega)$ vary smoothly. Evidently, we have

$$
\lim _{\omega \rightarrow 0+} R_{l}=\frac{p_{1} \omega+o(\omega)}{p_{2} \omega^{2}+o(\omega)}=+\infty,
$$

for $l \geq 2$, which implies that the arguments $\theta_{l}(\omega)$ start at $\pi / 2$ when $\omega \rightarrow 0+$.

By investigating the locus of $F_{6}(\omega)$ on a complex plane, we have found that the value of the argument $\theta_{6}(\omega)$ first falls from $\pi / 2$, tending to 0 ; as the trajectory of $F_{6}(\omega)$ passes from the first quadrant to the fourth, $\theta_{6}(\omega)$ does not really turn negative but jumps to $2 \pi$ instead and then it falls again, without second "jump" to perform. Similar phenomena occur in the other high-order systems when $l<$ 6 ; in each system, the argument $\theta_{l}(\omega)$ performs a jump at most once and no jumps for some of these systems, such as the second-order system, because the real and imaginary parts of $F_{2}(\omega)$ are always positive when $\omega>0$. The trajectories of $F_{2}(\omega)$ and $F_{6}(\omega)$ on the complex plane are shown in Figure 1 to give evidence of the variation on their arguments. For those whose arguments perform jumps, we consider the value of each $\theta_{l}(\omega)$ in two continuous intervals: $(0, \pi / 2)$ and $(0,2 \pi]$.

For $l=2,3$, if $\omega>\sqrt{\lambda_{n} / p_{2}}$ then $\operatorname{Re}\left[F_{l}(\omega)\right]=p_{2} \omega^{2}>\lambda_{n}$ and $M_{l}(\omega)>\lambda_{n}$, which we do not want. Thus, $\theta_{l}\left(\sqrt{\lambda_{n} / p_{2}}\right)$ is the minimum to consider. Then $p_{1} \omega-p_{3} \omega^{3}>0$ holds when $l=3$, which implies $\theta_{3}(\omega) \in(0, \pi / 2)$. Setting all $\tau_{m}<\bar{\tau}=$ $T_{l}\left(\sqrt{\lambda_{n} / p_{2}}\right)$, we have

$$
\omega \tau_{m}<\sqrt{\frac{\lambda_{n}}{p_{2}}} T_{l}\left(\sqrt{\frac{\lambda_{n}}{p_{2}}}\right)=\theta_{l}\left(\sqrt{\frac{\lambda_{n}}{p_{2}}}\right)<\theta_{l}(\omega),
$$

which contradicts (30); then by the same idea of the proof for the first-order system, the theorem for $l=2,3$ is proven.

Investigate the following parabola when $\omega \in\left[\sqrt{2 \lambda_{n} / p_{2}}\right.$, $\left.\sqrt{p_{2} / p_{4}-2 \lambda_{n} / p_{2}}\right]$ :

$$
\xi(\omega)=-p_{4} \omega^{4}+p_{2} \omega^{2}-\lambda_{n}
$$

Apparently, its maximal value comes at the point when $\omega=\sqrt{p_{2} / 2 p_{4}}$. According to the symmetry of parabolas, $\xi\left(\sqrt{2 \lambda_{n} / p_{2}}\right)=\xi\left(\sqrt{p_{2} / p_{4}-2 \lambda_{n} / p_{2}}\right)$ are the minima of $\xi(\omega)$. According to Assumption 3 that $p_{2} / p_{4}>3 p_{1} / p_{3}>6 \lambda_{n} / p_{2}$, which implies $p_{2}^{2}>4 \lambda_{n} p_{4}$, we have

$$
\begin{aligned}
\xi\left(\sqrt{2 \frac{\lambda_{n}}{p_{2}}}\right) & =2 \lambda_{n}-4 p_{4} \frac{\lambda_{n}^{2}}{p_{2}^{2}}-\lambda_{n}=\frac{\lambda_{n}}{p_{2}^{2}}\left(p_{2}^{2}-4 \lambda_{n} p_{4}\right) \\
& >0 .
\end{aligned}
$$

Thus, $\xi(\omega) \geq \xi\left(\sqrt{2 \lambda_{n} / p_{2}}\right)>0$. Then we have $M_{l}(\omega) \geq$ $\operatorname{Re}\left[F_{l}(\omega)\right] \geq p_{2} \omega^{2}-p_{4} \omega^{4}>\lambda_{n}$ for $l \geq 4$, when $\omega \in\left[\sqrt{2 \lambda_{n} / p_{2}}\right.$, $\left.\sqrt{p_{2} / p_{4}-2 \lambda_{n} / p_{2}}\right]$.

Let $F_{l}\left(\omega_{0}\right)$ be the first contact of its trajectory from the first quadrant to the fourth, as is shown in Figure 1. We analyze $\omega$ 
in two intervals: $\left(0, \omega_{0}\right)$ and $\left[\omega_{0},+\infty\right)$. Obviously, $\omega_{0}$ is the smallest positive real root of $\operatorname{Im}\left[F_{l}\left(\omega_{0}\right)\right]=0$.

Consider the interval $\omega<\omega_{0}$. If $\omega_{0} \in\left(\sqrt{2 \lambda_{n} / p_{2}}\right.$, $\left.\sqrt{p_{2} / p_{4}-2 \lambda_{n} / p_{2}}\right)$, then $\theta_{l}\left(\sqrt{2 \lambda_{n} / p_{2}}\right)$ would be smaller than the minimal possible value of $\theta_{l}(\omega)$ to satisfy (27), because $M(\omega)>\lambda_{n}$ if $\sqrt{2 \lambda_{n} / p_{2}}<\omega<\omega_{0}$.

Taking the fourth-order system into account, we have

$$
\omega_{0}=\sqrt{\frac{p_{1}}{p_{3}}} \in\left(\sqrt{\frac{2 \lambda_{n}}{p_{2}}}, \sqrt{\frac{p_{2}}{p_{4}}-\frac{2 \lambda_{n}}{p_{2}}}\right) .
$$

According to (16), one can obtain

$$
\begin{aligned}
\mid \operatorname{Im} & {\left[F_{4}\left(\sqrt{\frac{p_{2}}{p_{4}}-2 \frac{\lambda_{n}}{p_{2}}}\right)\right] \mid } \\
& =p_{3} \sqrt{\frac{p_{2}}{p_{4}}-2 \frac{\lambda_{n}}{p_{2}}}\left(\frac{p_{2}}{p_{4}}-2 \frac{\lambda_{n}}{p_{2}}-\frac{p_{1}}{p_{3}}\right) \\
> & p_{3} \sqrt{\frac{p_{1}}{p_{3}}}\left(\frac{p_{2}}{p_{4}}-2 \frac{p_{1}}{p_{3}}\right)=\sqrt{p_{3} p_{1}}\left(\frac{p_{2}}{p_{4}}-2 \frac{p_{1}}{p_{3}}\right) \\
> & \lambda_{n},
\end{aligned}
$$

where $p_{4}=1$. Because $\left|\operatorname{Im}\left[F_{4}(\omega)\right]\right|=p_{3} \omega^{3}-p_{1} \omega$ is monotonically increasing when $\omega \geq \omega_{0}$, we have $\left|\operatorname{Im}\left[F_{4}(\omega)\right]\right|>$
$\left|\operatorname{Im}\left[F_{4}\left(\sqrt{p_{2} / p_{4}-2 \lambda_{n} / p_{2}}\right)\right]\right|>\lambda_{n}$ for $\omega>\sqrt{p_{2} / p_{4}-2 \lambda_{n} / p_{2}}$. Then we know that $M_{4}(\omega)>\lambda_{n}$ holds if $\omega>\sqrt{2 \lambda_{n} / p_{2}}$. The only situation that needs to be considered is $\omega \leq \sqrt{2 \lambda_{n} / p_{2}}$, where $\theta_{l}\left(\sqrt{2 \lambda_{n} / p_{2}}\right)$ is the smallest value of $\theta_{l}(\omega)$; if we set all $\tau_{m}<\bar{\tau}=T_{4}\left(\sqrt{2 \lambda_{n} / p_{2}}\right)$, it can be obtained that

$$
\omega \tau_{m}<\sqrt{\frac{2 \lambda_{n}}{p_{2}}} T_{l}\left(\sqrt{\frac{2 \lambda_{n}}{p_{2}}}\right)=\theta_{l}\left(\sqrt{\frac{2 \lambda_{n}}{p_{2}}}\right)<\theta_{l}(\omega),
$$

which brings about the impossibility of (30) and provides the fourth-order system with consensus achievement.

For $l=5,6, \operatorname{Im}\left[F_{l}(\omega)\right]=p_{1} \omega-p_{3} \omega^{3}+p_{5} \omega^{5}$, then

$$
\omega_{0}=\sqrt{\frac{p_{3}-\sqrt{p_{3}^{2}-4 p_{1} p_{5}}}{2 p_{5}}} .
$$

Comparing $\omega_{0}^{2}$ and $p_{2} / p_{4}-2 \lambda_{n} / p_{2}$, we have

$$
\begin{gathered}
\left(\frac{p_{2}}{p_{4}}-2 \frac{\lambda_{n}}{p_{2}}\right)-\left(\frac{p_{3}-\sqrt{p_{3}^{2}-4 p_{1} p_{5}}}{2 p_{5}}\right) \\
=\sqrt{\left(\frac{p_{3}}{2 p_{5}}\right)^{2}-\frac{p_{1}}{p_{5}}}-\left(\frac{p_{3}}{2 p_{5}}-\frac{p_{2}}{p_{4}}+2 \frac{\lambda_{n}}{p_{2}}\right) .
\end{gathered}
$$

According to (17) and (18), $4-p_{5} p_{2}^{2} / p_{1} p_{4}^{2}>0$, so that

$$
\begin{gathered}
{\left[\left(\frac{p_{3}}{2 p_{5}}\right)^{2}-\frac{p_{1}}{p_{5}}\right]-\left(\frac{p_{3}}{2 p_{5}}-\frac{p_{2}}{p_{4}}+2 \frac{\lambda_{n}}{p_{2}}\right)^{2}} \\
=\frac{p_{1}}{p_{5}}\left[\left(2 \frac{p_{2} p_{3}}{p_{1} p_{4}}-6\right)+4 \frac{p_{5} \lambda_{n}}{p_{1} p_{2}}\left(\frac{p_{2}}{p_{4}}-\frac{\lambda_{n}}{p_{2}}\right)+\left(4-\frac{p_{5} p_{2}^{2}}{p_{1} p_{4}^{2}}\right)+\left(1-2 \frac{p_{3} \lambda_{n}}{p_{1} p_{2}}\right)\right]>0
\end{gathered}
$$

under Assumption 3. Thus (42) is also positive, which means $\omega_{0}<\sqrt{p_{2} / p_{4}-2 \lambda_{n} / p_{2}}$. When $\omega<\omega_{0}$, if we set all $\tau_{m}<$ $T_{l}\left(\sqrt{2 \lambda_{n} / p_{2}}\right)$, for $l=5,6,(30)$ is impossible.

Consider the interval $\omega \geq \omega_{0}$. Evidently, when $\omega \geq$ $\sqrt{p_{3} / 2}>\sqrt{p_{2} / p_{4}},\left|\operatorname{Re}\left[F_{5}(\omega)\right]\right|=p_{4} \omega^{4}-p_{2} \omega^{2}$ is monotonically increasing, According to Assumption 3, we have $p_{2} p_{3}>18 \lambda_{n}$. Then

$$
\begin{aligned}
\left|\operatorname{Re}\left[F_{5}\left(\sqrt{\frac{p_{3}}{2}}\right)\right]\right| & =\frac{p_{4} p_{3}^{3}}{4}-\frac{p_{2} p_{3}}{2}>\frac{3 p_{2} p_{3}}{4}-\frac{p_{2} p_{3}}{2} \\
& =\frac{p_{2} p_{3}}{4}>\lambda_{n} .
\end{aligned}
$$

Therefore, all $\tau_{m}<T_{l}\left(\sqrt{p_{3} / 2}\right)$ would make (30) impossible when $\omega \geq \omega_{0}$. As is described in Theorem 6, if all $\tau_{m}<$ $\min \left\{T_{l}\left(\sqrt{2 \lambda_{n} / p_{2}}\right), T_{l}\left(\sqrt{p_{3} / 2}\right)\right\}$, the consensus problem of the fifth-order system is solved.
Likewise, for $l=6$, according to (18) we have

$$
\begin{aligned}
\operatorname{Re} & {\left[F_{6}\left(\sqrt{\frac{p_{4}}{3}}\right)\right]-\lambda_{n} } \\
& =\sqrt{\frac{p_{4}}{3}}\left[p_{1}-p_{3} \frac{p_{4}}{3}+p_{5}\left(\frac{p_{4}}{3}\right)^{2}\right]-\lambda_{n} \\
& =\left(\sqrt{\frac{p_{4}}{3}}\right)^{3}\left[\frac{1}{3}\left(p_{5} p_{4}-3 p_{3}\right)\right]+\left(p_{1} \sqrt{\frac{p_{4}}{3}}-\lambda_{n}\right) \\
& >0
\end{aligned}
$$

when $\omega>\sqrt{p_{4} / 3}>\sqrt{p_{3} / p_{5}}, \operatorname{Re}\left[F_{6}(\omega)\right]$ is a monotonically increasing function. Since $\operatorname{Re}\left[F_{6}\left(\sqrt{p_{4} / 3}\right)\right]>\lambda_{n}$ is positive already, $M_{6}(\omega)$ would be further greater than $\lambda_{n}$ as the growth of $\omega$ has passed through $\sqrt{p_{4} / 3}$ where the situation should be ignored. Consequently, if all $\tau_{m}<$ $\min \left\{T_{l}\left(\sqrt{2 \lambda_{n} / p_{2}}\right), T_{l}\left(\sqrt{p_{4} / 3}\right)\right\}$, the sixth-order system is stable, and the theorem is proven out. 

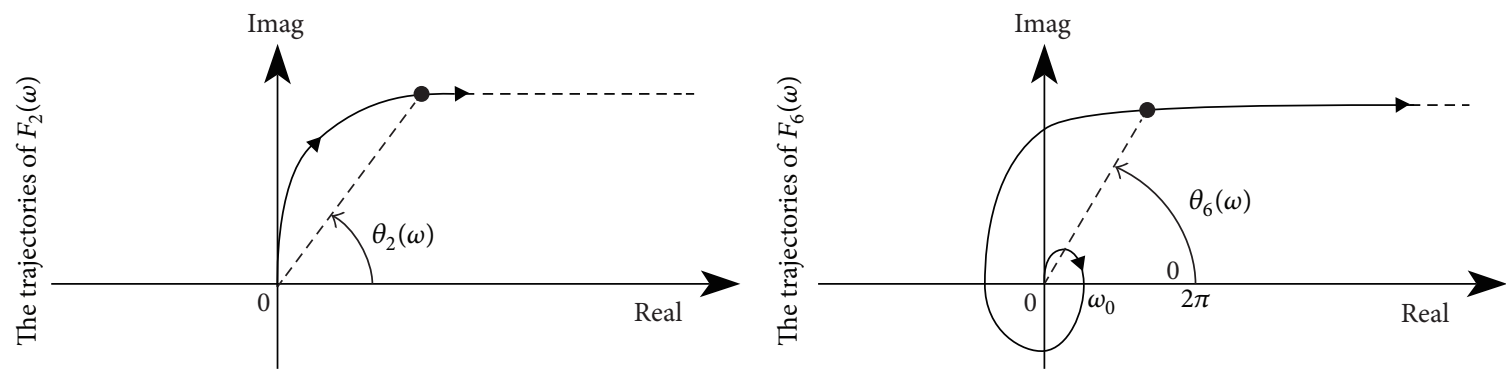

FIgURE 1: The trajectories of $F_{2}(\omega)$ and $F_{6}(\omega)$ on the complex plane.

Remark 7. Despite the fact that it is difficult to present a general solution in the form of inequality to consensus problems of all high-order multiagent systems because the monotonicity of each high-degree polynomial that the solution relies on requires specialized derivation to figure out, the process of deriving the consensus conditions for the firstto-sixth-order systems has demonstrated a general approach, with which one can work out consensus conditions for an arbitrary-order system. Moreover, by further calculation, we provide stronger consensus conditions for the first-order and second-order multiagent systems in the following theorem.

Theorem 8. Consider the first-order and second-order multiagent system (5) with the control input (3) $(l=1,2)$; then, the systems reach consensus if all the delays $\tau_{m}<\bar{\tau}_{l}$, where

$$
\begin{aligned}
\bar{\tau}_{1} & =\frac{\pi}{2 \lambda_{n}} \\
\bar{\tau}_{2} & =\sqrt{\frac{2}{\sqrt{p_{1}^{4}+4 \lambda_{n}^{2}}-p_{1}^{2}}} \\
& \cdot \arctan \left(\sqrt{\frac{2 p_{1}^{2}}{\sqrt{p_{1}^{4}+4 \lambda_{n}^{2}}-p_{1}^{2}}}\right) .
\end{aligned}
$$

Moreover, if all the delays $\tau_{m} \geq \bar{\tau}_{l}$, the systems will be unstable, and the agents will fail to reach consensus.

Proof. For the first-order multiagent system, we have already proven in Theorem 6 that $\tau_{m}<\bar{\tau}_{1}$ is a sufficient condition for the system to reach consensus. If all $\tau_{m}=\bar{\tau}_{1}, j \lambda_{n}$ is the imaginary eigenvalue and its corresponding eigenvector $u$ is the one ensuring $\left|\sum_{m=1}^{M} \alpha_{m}\right|=\lambda_{n} \triangleq \bar{\omega}_{1}$. With these values substituted into (27) $(l=1)$, the equation

$$
\sum_{m=1}^{M} \alpha_{m} e^{j \bar{\omega}_{1} \bar{\tau}_{1}}=F_{1}\left(\bar{\omega}_{1}\right)
$$

holds true; that is, $\bar{\tau}_{1}$ is the "delay margin" equal to which the delays of the first-order system present the first contact of the eigenvalues from the stable region to the unstable one. When all the delays exceed the delay margin, the systems will be unstable, because there then exist eigenvalues in the unstable region (the RHP). The instabilities become stronger and the delays get greater distances exceeding the delay margin.
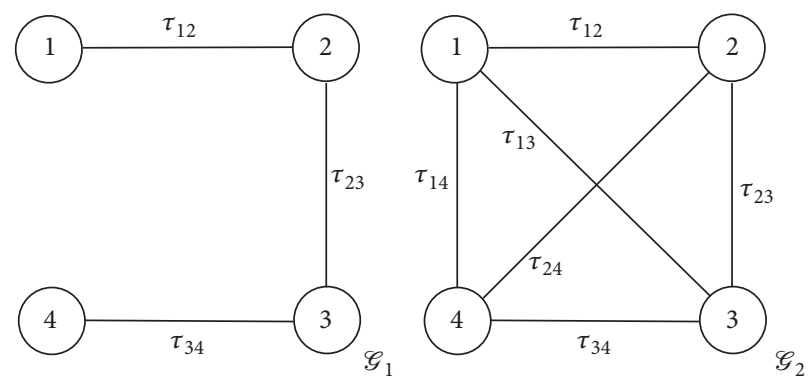

FIgURE 2: Two communication topologies of the 4-agent system.

For $l=2, M_{2}(\omega)=\sqrt{\omega^{4}+p_{1}^{2} \omega^{2}}$. Let $\omega^{*}=$ $\sqrt{\left(\sqrt{p_{1}^{4}+4 \lambda_{n}^{2}}-p_{1}^{2}\right) / 2}$ be the only positive root of $M_{2}(\omega)=$ $\lambda_{n}$; thus $M_{2}(\omega) \leq \lambda_{n}$ as long as $\omega \leq \omega^{*}$. Owing to the decline of $\theta(\omega)$, we can set all $\tau_{m}<\theta\left(\omega^{*}\right) / \omega^{*}=\bar{\tau}_{2}$ to avoid the possibility of (27) $(l=2)$. Therefore the sufficiency of the condition has been proven. By the same principle as that for the first-order system, $\bar{\tau}_{2}$ is also the delay margin bringing about the failure of the system to reach consensus.

\section{Numerical Simulations}

In this section, some simulations are provided to illustrate the theoretical results obtained by the previous analysis.

Consider a multiagent system consisting of 4 agents. Figure 2 shows two different communication topologies described with undirected graphs $\mathscr{G}_{1}$ and $\mathscr{G}_{2}$, respectively. With three different time delays on each connection, $\mathscr{G}_{1}$ presents the simplest connected topology, while $\mathscr{G}_{2}$ having six different time delays displays full connectivity. All the delays are marked with $\tau_{i j}$, where $i$ and $j$ are the indices of the connected agents. Suppose the weight of each edge on both graphs is 1 ; then, for $\mathscr{G}_{1}, \lambda_{n} \approx 3.4142$, and, for $\mathscr{G}_{2}$, $\lambda_{n}=4$. Selected experiments will be carried out to validate the obtained results on 4 -agent systems with both topologies illustrated by $\mathscr{G}_{1}$ and $\mathscr{G}_{2}$.

For a third-order multiagent system with simply connected graph $\mathscr{G}_{1}$, set $p_{1}=9$ and $p_{2}=6$; thus $\bar{\tau}=1.43$ according to Theorem 6 . Let $\tau_{12}=1.42, \tau_{23}=1.40$, and $\tau_{34}=$ 1.38. Seen from the simulation results shown in Figure 3, it is apparent that agents have reached consensus. And for the system with topology $\mathscr{G}_{2}$ showing full connectivity, let $p_{1}=9$ 

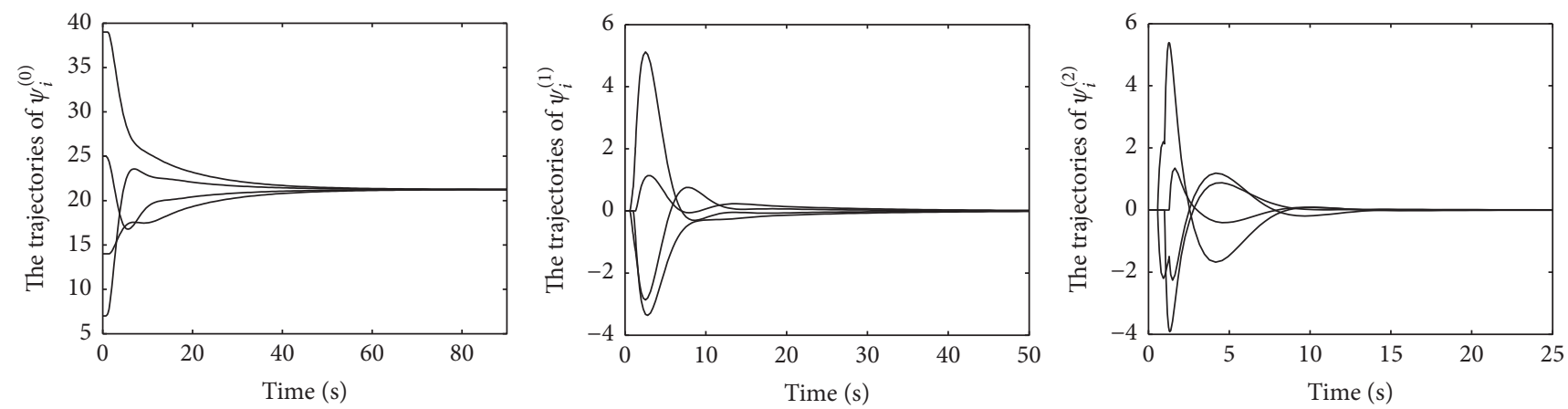

FIGURE 3: The trajectories of agents in the third-order system (the topology is $\mathscr{G}_{1}$ ).
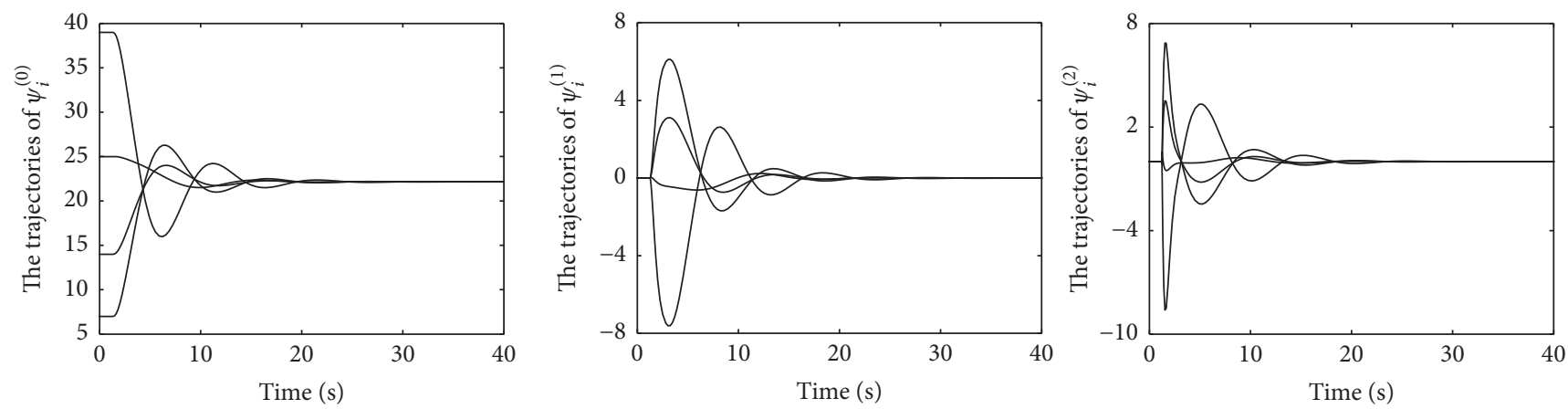

FIGURE 4: The trajectories of agents in the third-order system (the topology is $\mathscr{G}_{2}$ ).
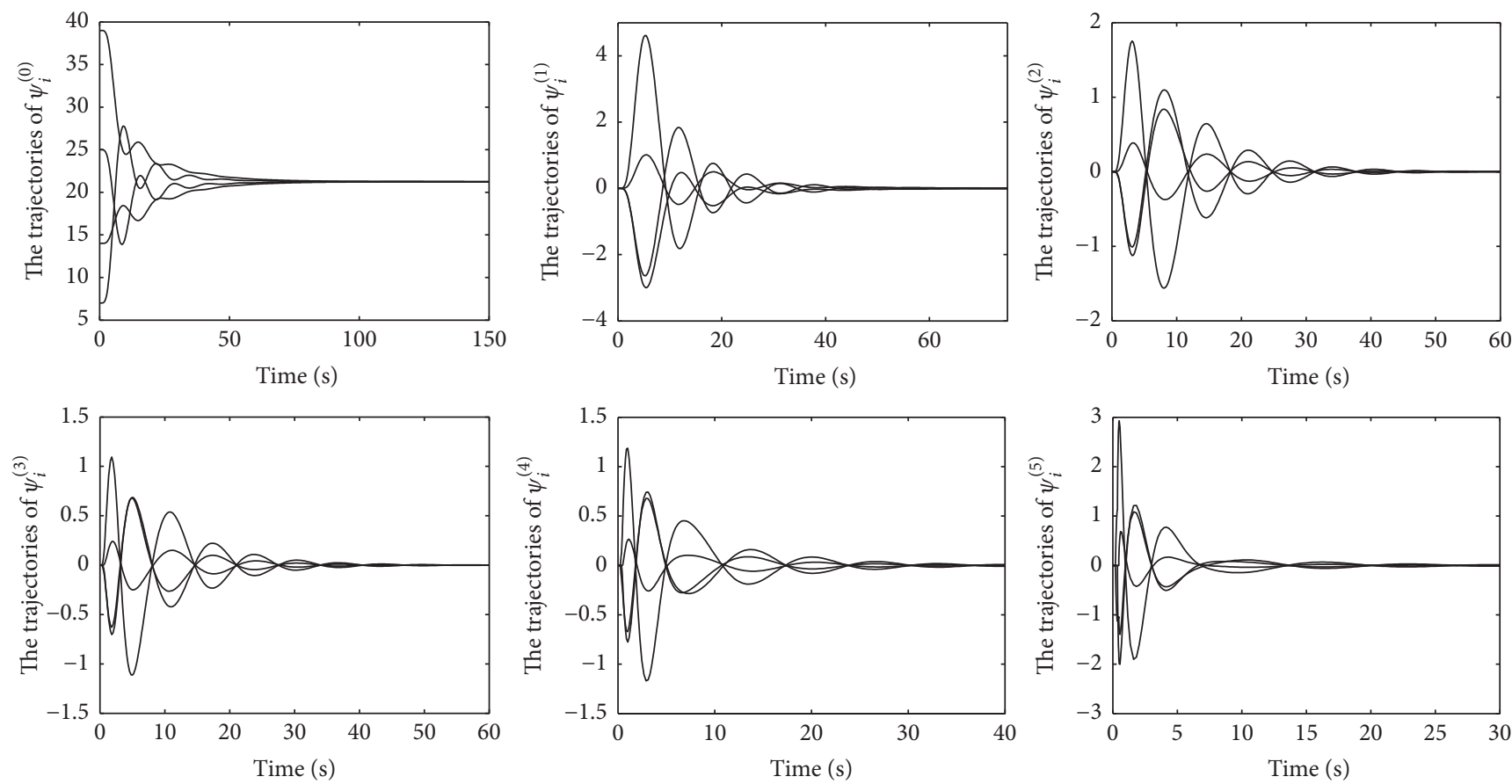

FIGURE 5: The trajectories of agents in the sixth-order system (the topology is $\mathscr{G}_{1}$ ). 

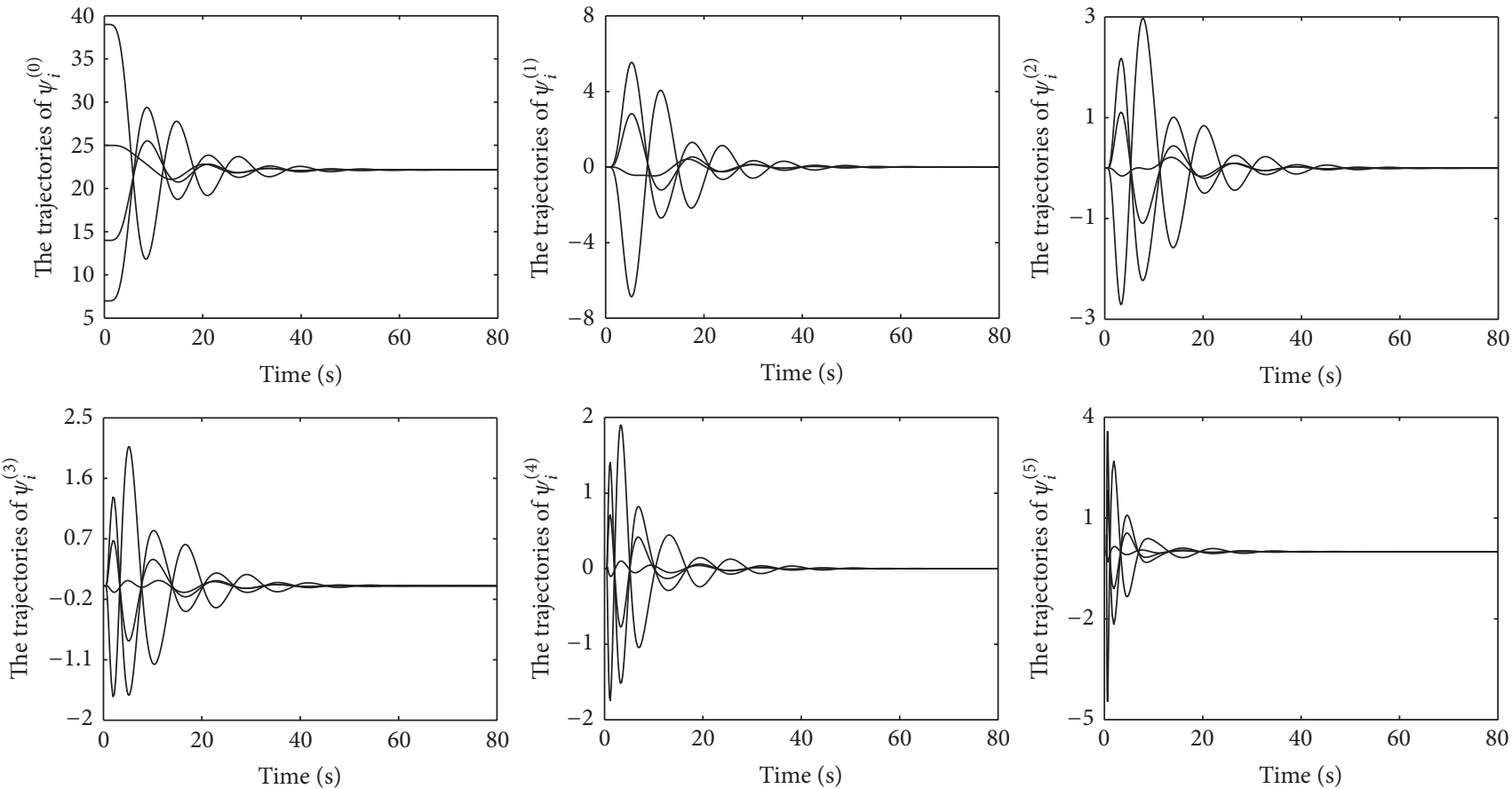

FIGURE 6: The trajectories of agents in the sixth-order system (the topology is $\mathscr{G}_{2}$ ).
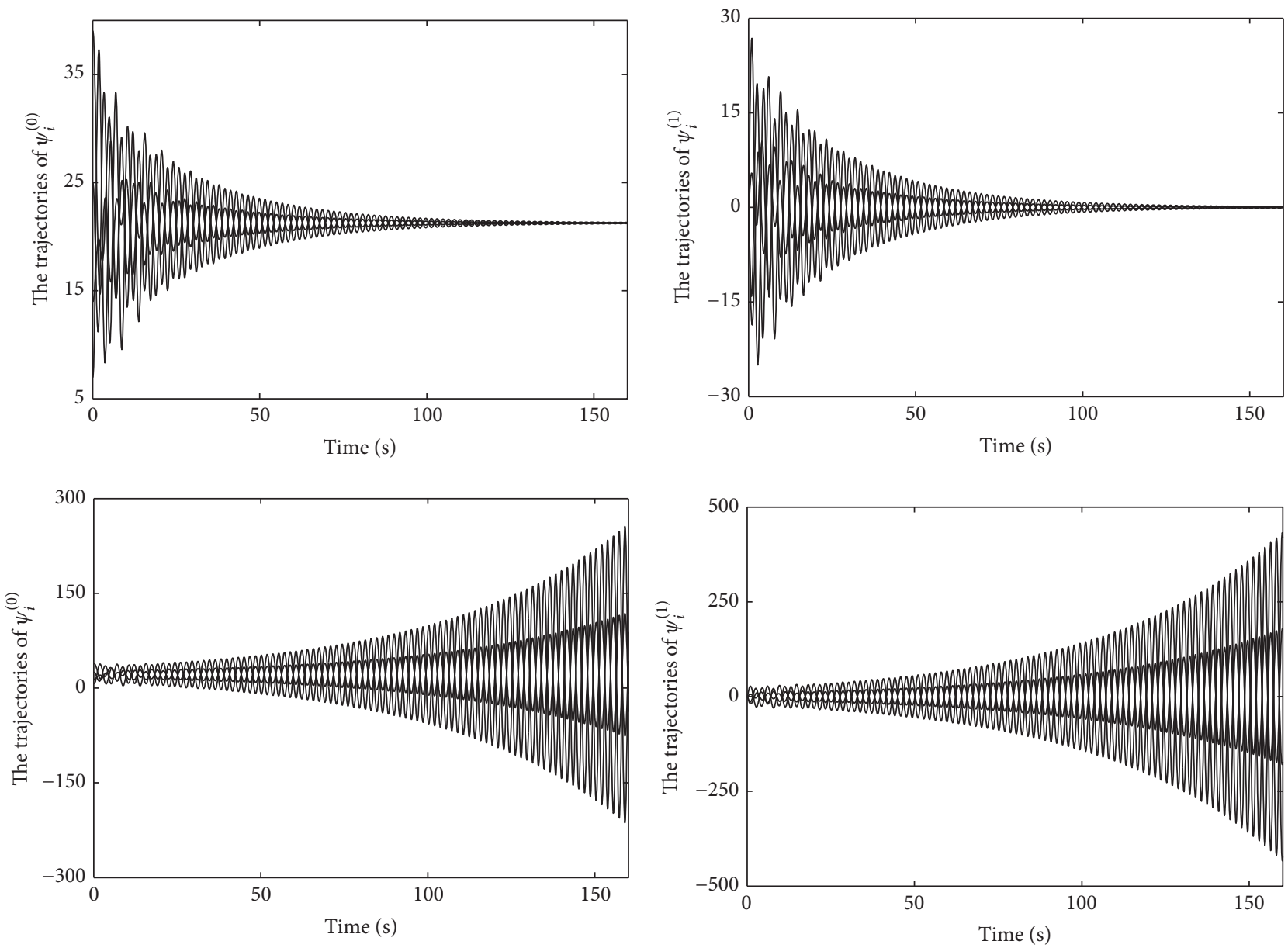

Figure 7: The trajectories of agents in the second-order system (the topology is $\mathscr{G}_{1}$ ). 

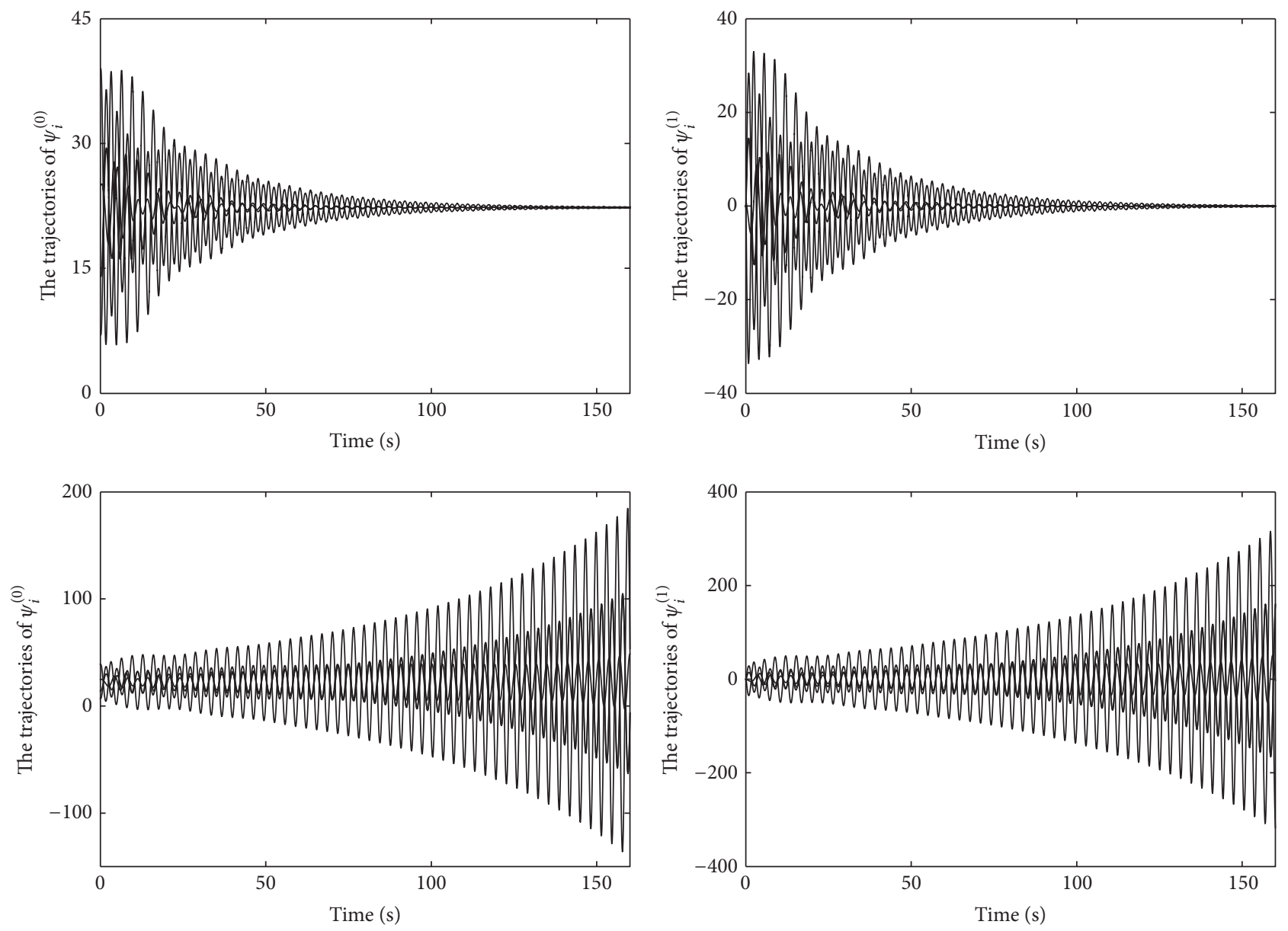

FIGURE 8: The trajectories of agents in the second-order system (the topology is $\mathscr{G}_{2}$ ).

and $p_{2}=6$, and then one gets $\bar{\tau}=1.2729$. With time delays $\tau_{12}=1.27, \tau_{23}=1.26, \tau_{34}=1.25, \tau_{14}=1.24, \tau_{13}=1.23$, and $\tau_{24}=1.22$, simulation results are depicted in Figure 4 , where the consensus achievement recurs.

For a sixth-order system connected as $\mathscr{G}_{1}$, the parameters $p_{1}=12, p_{2}=27, p_{3}=40, p_{4}=28$, and $p_{5}=12$ yield $\bar{\tau}=0.51$. Figure 5 has shown that the consensus achievement is guaranteed, where $\tau_{12}=0.50, \tau_{23}=0.48$, and $\tau_{34}=0.46$. With connections in $\mathscr{G}_{2}$, the system reaches consensus as shown in Figure 6, where $p_{1}=12, p_{2}=27, p_{3}=35, p_{4}=28$, and $p_{5}=12$ and time delays $\tau_{12}=0.47, \tau_{23}=0.46, \tau_{34}=0.45$, $\tau_{14}=0.44, \tau_{13}=0.43$, and $\tau_{24}=0.42$ which are bounded by $\bar{\tau}=0.4713$ calculated according to Theorem 6 .

To examine Theorem 8 , we set $p_{1}=0.4$ for the secondorder system. According to Theorem 8 , for $\mathscr{G}_{1}$, the delay margin $\bar{\tau}=0.1181$. Two groups of delays are set: $\tau_{12}=$ $0.11, \tau_{23}=0.10, \tau_{34}=0.09$, which are bounded by the delay margin, and $\tau_{12}=0.12, \tau_{23}=0.13, \tau_{34}=0.14$, which exceed the delay margin. The results are shown in Figure 7: the first two figures are the trajectories of agents when time delays are bounded, which indicates that and consensus is reached; the last two figures are the trajectories of agents when all time delays exceed the delay margin, where the system is unstable. These phenomena have attested the theorem. And for $\mathscr{G}_{2}$, similar experiment is carried out: the delay margin is obtained as $\bar{\tau}=0.1007$, and then, respectively, gives the bounded delays $\tau_{12}=0.10, \tau_{23}=0.09, \tau_{34}=0.08, \tau_{14}=$ $0.07, \tau_{13}=0.06$, and $\tau_{24}=0.05$ and the exceeded delays $\tau_{12}=0.101, \tau_{23}=0.104, \tau_{34}=0.107, \tau_{14}=0.11, \tau_{13}=0.113$, and $\tau_{24}=0.116$. As shown in Figure 8 , the simulation results validate the theorem again.

\section{Conclusions}

This paper has studied consensus problem of high-order multiagent systems with nearest-neighbor control rules in the presence of nonuniform time delays. For each delayed $l$ th-order $(l=1,2, \ldots, 6)$ system, a sufficient condition has been provided in the form of inequalities and, for the first-order and second-order system, consensus conditions have been presented in the form of delay margins, which are less conservative and simpler in calculation than the existing results by Lyapunov methods in the form of LMIs. Numerical simulations on systems with two sets of different topologies have been carried out to testify the theorems. The simulation results show that the selected experiments have 
reached expected effect: the systems achieve consensus under given conditions.

Future research will seek solutions to consensus problems of nonuniformly delayed high-order systems with directed topologies by applying this method. The main challenge is the calculation of $F_{l}(\omega)$ 's arguments (see (27)), because the eigenvalues of systems with directed topologies are complex values even in the absence of time delays and it is hard to tell the relationship among the arguments of several complex values and their summation.

\section{Competing Interests}

The authors declare that they have no competing interests.

\section{Acknowledgments}

This work has been supported by the Specialized Research Fund for the Doctoral Program of Higher Education (20130185110023).

\section{References}

[1] Z. Li, G. Wen, Z. Duan, and W. Ren, "Designing fully distributed consensus protocols for linear multi-agent systems with directed graphs," IEEE Transactions on Automatic Control, vol. 60, no. 4, pp. 1152-1157, 2015.

[2] Z. Li, W. Ren, X. Liu, and M. Fu, "Consensus of multi-agent systems with general linear and lipschitz nonlinear dynamics using distributed adaptive protocols," IEEE Transactions on Automatic Control, vol. 58, no. 7, pp. 1786-1791, 2013.

[3] G. Zhang, J. Xu, J. Zeng, J. Xi, and W. Tang, "Consensus of high-order discrete-time linear networked multi-agent systems with switching topology and time delays," Transactions of the Institute of Measurement and Control, 2016.

[4] C. Sun, G. Hu, and L. Xie, "Robust consensus tracking for a class of high-order multi-agent systems," International Journal of Robust and Nonlinear Control, vol. 26, no. 3, pp. 578-598, 2016.

[5] X. Dong, Z. Shi, G. Lu, and Y. Zhong, “Time-varying output formation control for high-order linear time-invariant swarm systems," Information Sciences, vol. 298, pp. 36-52, 2015.

[6] C.-C. Hua, X. You, and X.-P. Guan, "Leader-following consensus for a class of high-order nonlinear multi-agent systems," Automatica, vol. 73, pp. 138-144, 2016.

[7] W. Ren, K. Moore, and Y.-Q. Chen, "High-order consensus algorithms in cooperative vehicle systems," in Proceedings of the IEEE International Conference on Networking, Sensing and Control (ICNSC '06), pp. 457-462, Ft. Lauderdale, Fla, USA, April 2006.

[8] P. Lin, Z. Li, Y. Jia, and M. Sun, "High-order multi-agent consensus with dynamically changing topologies and timedelays," IET Control Theory and Applications, vol. 5, no. 8, pp. 976-981, 2011.

[9] Q.-J. Zhang, Y.-F. Niu, L. Wang, L.-C. Shen, and H.-Y. Zhu, "Average consensus seeking of high-order continuous-time multi-agent systems with multiple time-varying communication delays," International Journal of Control, Automation and Systems, vol. 9, no. 6, pp. 1209-1218, 2011.
[10] G. Wen, Z. Duan, W. Yu, and G. Chen, "Consensus of secondorder multi-agent systems with delayed nonlinear dynamics and intermittent communications," International Journal of Control, vol. 86, no. 2, pp. 322-331, 2013.

[11] P. Lin and Y. Jia, "Multi-agent consensus with diverse timedelays and jointly-connected topologies," Automatica, vol. 47, no. 4, pp. 848-856, 2011.

[12] W. Yu, G. Chen, M. Cao, and W. Ren, "Delay-induced consensus and quasi-consensus in multi-agent dynamical systems," IEEE Transactions on Circuits and Systems I: Regular Papers, vol. 60, no. 10, pp. 2679-2687, 2013.

[13] Y.-P. Tian and C.-L. Liu, "Robust consensus of multi-agent systems with diverse input delays and asymmetric interconnection perturbations," Automatica, vol. 45, no. 5, pp. 1347-1353, 2009.

[14] P.-A. Bliman and G. Ferrari-Trecate, "Average consensus problems in networks of agents with delayed communications," Automatica, vol. 44, no. 8, pp. 1985-1995, 2008.

[15] Y.-P. Tian and C.-L. Liu, "Consensus of multi-agent systems with diverse input and communication delays," IEEE Transactions on Automatic Control, vol. 53, no. 9, pp. 2122-2128, 2008.

[16] P. Lin, Y.-M. Jia, J.-P. Du, and S.-Y. Yuan, "Distributed consensus control for second-order agents with fixed topology and timedelay," in Proceedings of the 26th IEEE Chinese Control Conference (CCC '07), pp. 577-581, Zhangjiajie, China, July 2007.

[17] P. Lin and Y. Jia, "Consensus of second-order discrete-time multi-agent systems with nonuniform time-delays and dynamically changing topologies," Automatica, vol. 45, no. 9, pp. 21542158, 2009.

[18] V. D. Blondel, J. M. Hendrickx, A. Olshevsky, and J. N. Tsitsiklis, "Convergence in multiagent coordination, consensus, and flocking," in Proceedings of the 44th IEEE Conference on Decision and Control and European Control Conference (CDCECC '05), pp. 2996-3000, IEEE, 2005.

[19] U. Munz, A. Papachristodoulou, and F. Allgower, "Generalized Nyquist consensus condition for high-order linear multi-agent systems with communication delays," in Proceedings of the 48th IEEE Conference on Decision and Control, Held Jointly with the 28th Chinese Control Conference (CDC/CCC '09), pp. 47654771, IEEE, Shanghai, China, December 2009.

[20] U. Munz, A. Papachristodoulou, and F. Allgower, "Delay robustness in non-identical multi-agent systems," IEEE Transactions on Automatic Control, vol. 57, no. 6, pp. 1597-1603, 2012.

[21] A. Popov and H. Werner, "Robust stability of a multiagent system under arbitrary and time-varying communication topologies and communication delays," IEEE Transactions on Automatic Control, vol. 57, no. 9, pp. 2343-2347, 2012.

[22] H. Fang, Z. Wu, and J. Wei, "Improvement for consensus performance of multi-agent systems based on weighted average prediction," IEEE Transactions on Automatic Control, vol. 57, no. 1, pp. 249-254, 2012.

[23] P. Lin, K.-Y. Qin, Z.-K. Li, and W. Ren, "Collective rotating motions of second-order multi-agent systems in threedimensional space," Systems \& Control Letters, vol. 60, no. 6, pp. 365-372, 2011.

[24] B. Mirkin, P.-O. Gutman, and Y. Shtessel, "Asymptotic sliding mode control approach to adaptive distributed tracking problem for multi-agent nonlinear delayed systems," International Journal of Control, vol. 85, no. 11, pp. 1671-1682, 2012.

[25] P. Deshpande, P. P. Menon, C. Edwards, and I. Postlethwaite, "Formation control of multi-agent systems with double integrator dynamics using delayed static output feedback," in 
Proceedings of the 50th IEEE Conference on Decision and Control and European Control Conference (CDC-ECC '11), pp. 34463451, Orlando, Fla, USA, December 2011.

[26] H. Zhang, J. Zhang, G. H. Yang, and Y. Luo, "Leader-based optimal coordination control for the consensus problem of multiagent differential games via fuzzy adaptive dynamic programming," IEEE Transactions on Fuzzy Systems, vol. 23, no. 1, pp. 152-163, 2015.

[27] T. H. Lee, J. H. Park, D. H. Ji, and H. Y. Jung, "Leader-following consensus problem of heterogeneous multi-agent systems with nonlinear dynamics using fuzzy disturbance observer," Complexity, vol. 19, no. 4, pp. 20-31, 2014.

[28] R. Ponalagusamy and S. Senthilkumar, "Investigation on numerical solution for a robot arm problem," Journal of Automation Mobile Robotics and Intelligent Systems, vol. 3, pp. 34-40, 2009.

[29] C. P. Chen, G.-X. Wen, Y.-J. Liu, and Z. Liu, "Observer-based adaptive backstepping consensus tracking control for highorder nonlinear semi-strict-feedback multiagent systems," IEEE Transactions on Cybernetics, vol. 46, no. 7, pp. 1591-1601, 2016.

[30] M. Shi and K. Qin, "Distributed control for multiagent consensus motions with nonuniform time delays," Mathematical Problems in Engineering, vol. 2016, Article ID 3567682, 10 pages, 2016.

[31] C. D. Godsil, G. Royle, and C. Godsil, Algebraic Graph Theory, vol. 8, Springer, New York, NY, USA, 2001.

[32] Y.-Y. Nie and X.-K. Xie, "New criteria for polynomial stability," IMA Journal of Mathematical Control and Information, vol. 4, no. 1, pp. 1-12, 1987. 


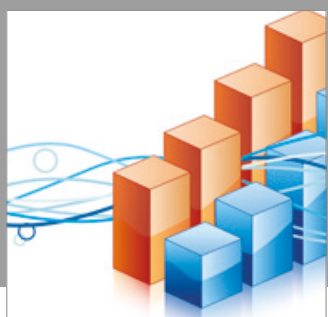

Advances in

Operations Research

vatersals

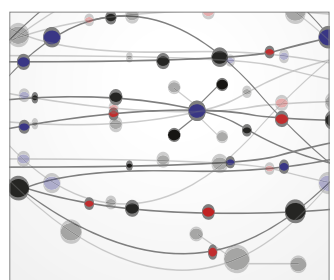

\section{The Scientific} World Journal
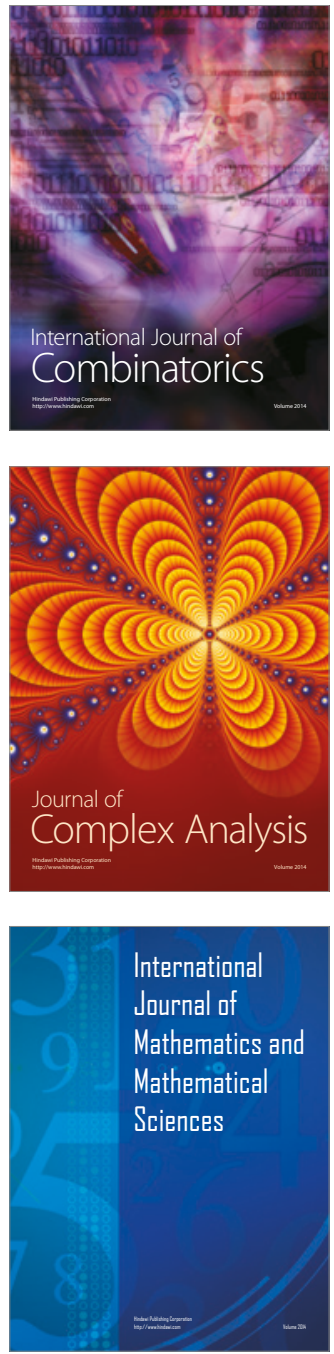
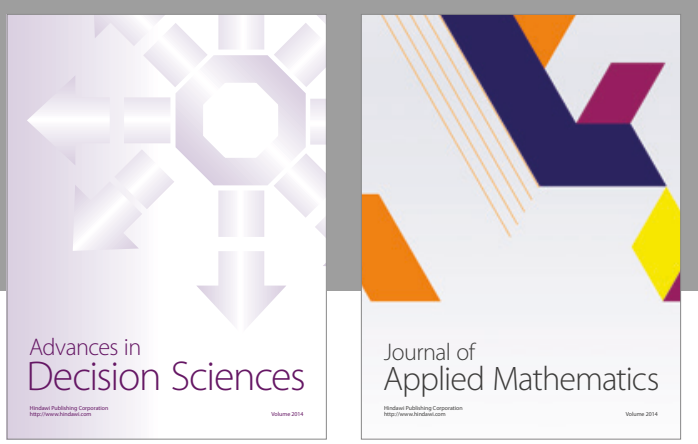

Algebra

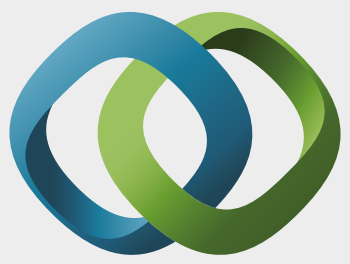

\section{Hindawi}

Submit your manuscripts at

https://www.hindawi.com
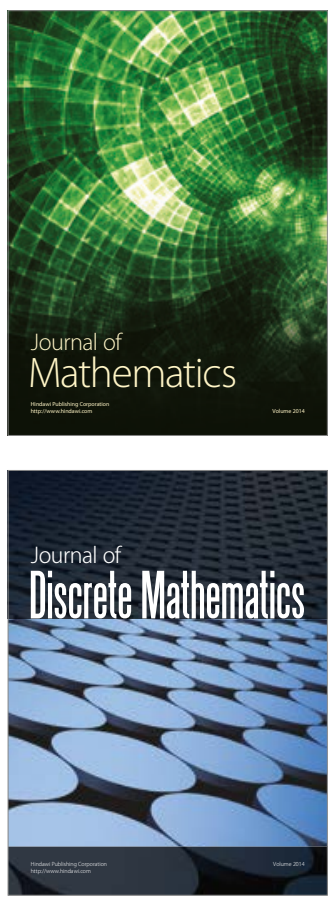

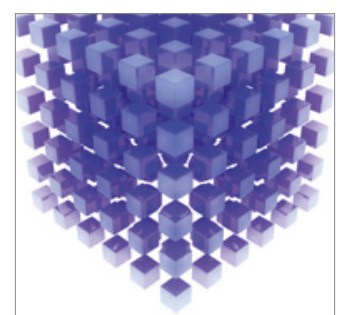

Mathematical Problems in Engineering
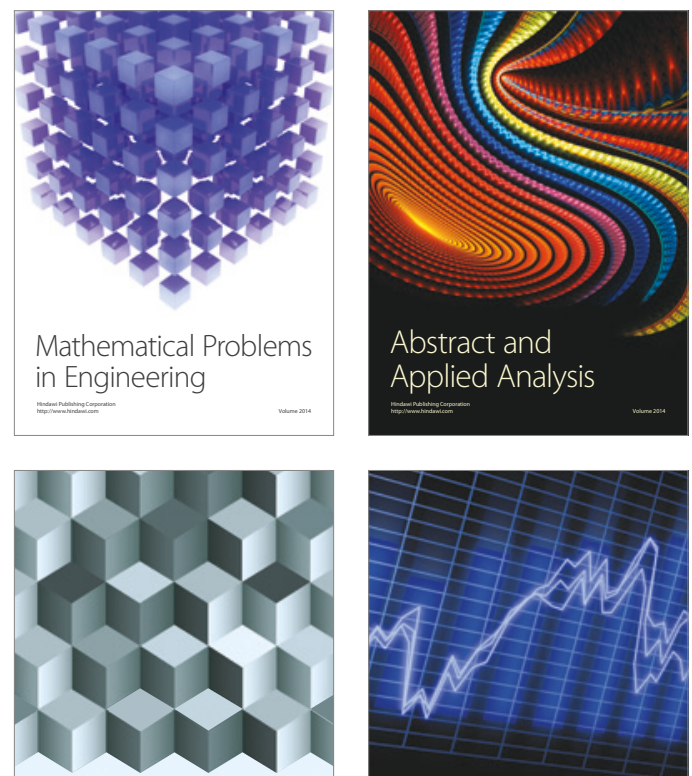

Journal of

Function Spaces

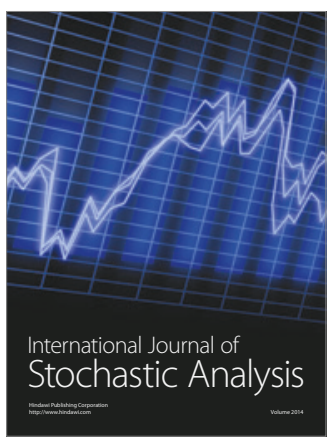

Probability and Statistics
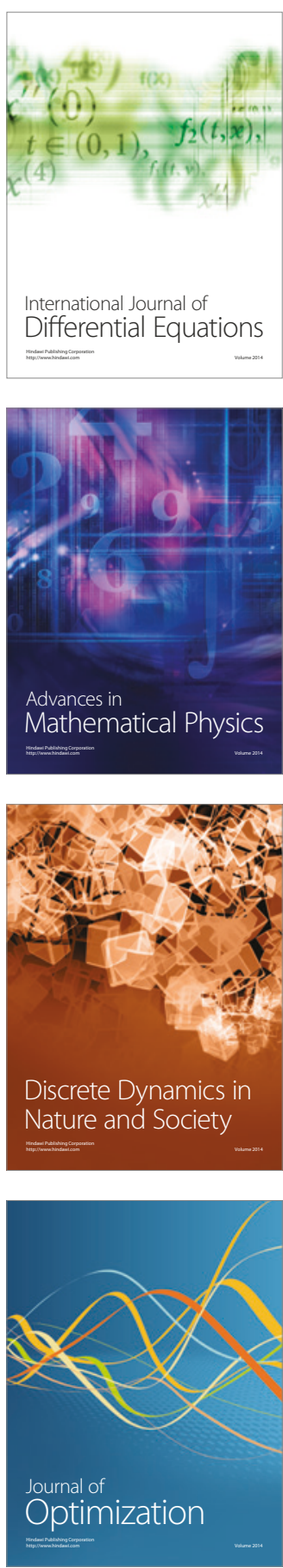